Stoa

Vol. 11 , no. 21,2020 , pp. 110-137

ISSN 2007-1868

\title{
EL PENSAMIENTO ANTROPOLÓGICO DE SCHELER Y SU PRONTA RECEPCIÓN EN ESPAÑA*
}

\author{
Íngrid Vendrell
}

\section{Los orígenes del pensamiento antropológico de Scheler}

No fue sino hasta la década de 1920 que el mundo de habla alemana comenzó a darse cuenta de que la antropología filosófica se había convertido en su propio campo temático y nueva disciplina. Autores como Scheler, Plessner, Pfänder, Verweyen y Gehlen han contribuido decisivamente a esta toma de conciencia. Especialmente, el texto de Max Scheler El puesto del hombre en el cosmos de 1928 se considera como el documento fundador de la antropología filosófica contemporánea. Sin embargo, el interés de Scheler en la pregunta, qué es el hombre, queda claro mucho antes. Ya en sus primeros textos, no solo en el trabajo posterior del período de Colonia de los años 20, la pregunta puede verse como una guía para su pensamiento. El mismo Scheler afirma: "Las preguntas: ¿qué es el hombre, y cuál es su posición de ser, han sido más importantes para mí desde el primer despertar de mi conciencia filosófica que cualquier otra cuestión filosófica? El problema se resumió en 1922 en la elaboración de una gran obra dedicada a esta cuestión, y tuve la suerte de ver que la mayoría de los problemas de la filosofía con los que ya he tratado coinciden cada vez más en esta cuestión."1 En qué medida este reclamo está motivado por la voluntad de Scheler de presentarse como el fundador

Recibido 12 de noviembre de 2019 Aceptado 23 de noviembre de 2019

\footnotetext{
* Traducido por Marisol Ramírez Patiño de Scheler anthropologisches Denken un die frühe Rezeption in Spanien. Phanomenologische Foschungen, Felix Meiner Verlag, 2009, pp. 175-202.

${ }^{1}$ Scheler, M., El puesto del hombre en el cosmos, en GW, IX, p. 9. Citado de Pintor Ramos, A., "la influencia de Scheler en el pensamiento del mundo de habla hispana" en Investigación fenomenológica, 28/29, 1993, pp. 314-331, aquí p. 314. Citaremos los escritos de Scheler por la edición de las Obras
} 
de la nueva disciplina y posicionarse frente a posibles rivales como Plessner, lo dejaré abierto aquí. Sin embargo, es obvio que los primeros textos de la fase "realista" de Scheler, y en particular sus contribuciones a la filosofía de los sentimientos entre 1910 y 1920, dan testimonio de este interés antropológico. Si bien los textos de esta fase no abordan directamente la cuestión de cómo es el hombre, tal y como hiciese en textos posteriores, se les puede llamar "antropológicos". Porque están preocupados, aunque en estado embrionario, con cuestiones fundamentales de esta disciplina y contienen en pocas palabras los desarrollos posteriores de la antropología filosófica de Scheler. En concreto encontramos que Scheler, en relación con la cuestión ética sobre la acción correcta, desarrolla su propia teoría de sensaciones y sentimientos y analiza toda una serie de fenómenos emocionales como el resentimiento, la simpatía, el remordimiento, la vergüenza y la humildad. Se presta especial atención al amor en este contexto. Común a los textos de esta fase se encuentra el postulado antropológico de que el hombre está determinado principalmente por su vida emocional, especialmente a través del amor como el núcleo de la personalidad y la posibilidad de acceso al mundo. ${ }^{2}$ La primordialidad del sentimiento antes del pensar y el actuar se representa claramente en este texto, y el hombre se concibe principalmente como un ser sensible. Está justificado hablar del "pensamiento antropológico" de Scheler, aunque no podemos hablar de "antropología filosófica" en el sentido más amplio de la palabra con la conciencia de una nueva disciplina. Estos primeros textos de Scheler hasta ahora han recibido poca atención desde el punto de vista de la antropología filosófica, aunque pueden abrir nuevas líneas de investigación fructíferas para la antropología. A este olvido ha contribuido el hecho que la disciplina mencionada se centró casi exclusivamente en el trabajo posterior de Scheler, en el que él mismo explota explícitamente la "antropología", dejando en una posición secundaria a los sentimientos. Una excepción, por supuesto, es la recepción del pensamiento filosófico de Scheler en España. La inclusión de las tesis de Scheler no tiene lugar aquí a través de su obra explícitamente antropológica de la época en Colonia, sino en sus primeros textos sobre el tema de los sentimientos, el sentir y el amor. Como me gustaría mostrar en lo que sigue, toda la recepción de Scheler en España articula este tema de modo que los textos de Scheler de la fase realista son el foco de atención particular en

Completas (Gesammelte Werke, Frankle-Bouvier Verlag, Berna-Bonn), señalando volumen y página (nota del traductor).

${ }^{2}$ Scheler, M. Ordo amoris, en GW, X, pp. 345-376. 
este país. Este enfoque especial en los sentimientos no se encuentra en otros países. Por ejemplo, la recepción de Scheler en Francia se centra en el tema de los valores, la persona y la intersubjetividad. ${ }^{3}$

En este contexto, surgen algunas preguntas. ¿Sobre qué canales funciona exactamente esta recepción? ¿Cómo tiene éxito la inclusión y el desarrollo del pensamiento antropológico de Scheler en España? En este ensayo, me gustaría argumentar que la recepción del pensamiento de Scheler en España se lleva a cabo de dos maneras. Por un lado, se deberán mencionar las traducciones y no solo las traducciones de los escritos de Scheler, sino también las de otros fenomenólogos. La traducción de las obras de Brentano, Husserl, Pfänder y Kolnai allanaron el camino para una recepción de Scheler, porque transmitieron todo el marco de discusión en el que se desarrollaron y despliegan las tesis del filósofo. El otro enfoque es la recepción y el desarrollo críticos de las ideas de Scheler por parte de filósofos e intelectuales españoles. Como los propios traductores de Scheler eran filósofos versados en fenomenología, a veces ambos caminos de recepción se superponen. Dicho desarrollo se articula en torno al tema de la afectividad humana: la capacidad humana para sentir, los valores percibidos y el amor como núcleo de la persona. También muestra la originalidad de los pensadores españoles que incorporan las ideas de Scheler en sus intereses antropológicos y los desarrollan en la dirección de su propia antropología fenomenológica.

La importancia de este postulado antropológico, en la cual el hombre se caracteriza principalmente por su capacidad de sentir, a menudo ha sido pasado por alto o subestimado en los pocos estudios sobre la influencia de Scheler en España. Esto tiene que ver con el hecho de que el tema de las emociones hasta ahora ha sido considerado como un tema que no es realmente importante para la filosofía. Pintor Ramos ha reconocido la importancia del amor como tema en su ensayo sobre la influencia de Scheler en el trabajo de Ortega, pero limita su análisis sólo a Ortega y Gasset. ${ }^{4}$ Su importancia para la recepción de Scheler es indudable, pero otros autores como García Morente o Joaquín Xirau con sus correspondientes concepciones de valores, sentir y sentimientos también juegan un papel importante. La importancia de estos otros autores para el posterior desarrollo de las tesis sobre el papel del sentimiento se ha

${ }^{3}$ Cfr. Leroux, H., "Algunos aspectos sobre la recepción de Max Scheler en Francia”, en Investigación fenomenológica., 28/29, 1993, pp. 332-356.

${ }^{4}$ Pintor Ramos ha señalado los tres hilos principales en la recepción de Scheler por parte de Ortega y Gasset: la teoría de la intersubjetividad, el concepto de valor y el amor. $C f$ r. Pintor Ramos, 1993, p. 319. 
ignorado hasta ahora. En el trabajo de San Martín Fenomenología en España, apenas se discute el significado de los textos de la fase realista de Scheler para los autores españoles. ${ }^{5}$ Veo mi trabajo como complementario a estos dos estudios y como un intento de llenar los vacíos mencionados. Me centraré en las tesis antropológicas de Scheler en 1910 y 1920 y en la primera fase de la recepción de los textos de Scheler en España. ${ }^{6}$ Por lo tanto, paso primero a los desarrollos históricos de este recibimiento del pensamiento de Scheler en España y luego al contenido y desarrollo de su pensamiento por parte de los autores españoles.

\section{La filosofía española a principios del sigloxx}

\subsection{Decadencia e impulsos de dinamización}

Se necesita una mirada a la filosofía española a principios del siglo XX para comprender mejor las formas de recepción de Scheler. La filosofía española de principios del siglo XX carece del vínculo con su pasado (León, Nebrija, Alpizcueta, Soto, Suárez) y carece de instalaciones como sociedades filosóficas y revistas. Hay una escasez de instituciones culturales y en las bibliotecas incluso faltan libros esenciales.$^{7}$ Desde el siglo XVIII, España había perdido su propia tradición intelectual. Por otro lado, la conexión con Europa apenas existía, por lo que las nuevas corrientes filosóficas apenas llegaron a España. ${ }^{8}$ Muchos autores a finales del siglo XIX describieron esta situación de deseo cultural con gran pesimismo, especialmente los autores de la llamada "Generación de 1898 ", a la que pertenecía Unamuno.

En la primera década del siglo y en la estela de Miguel de Unamuno y José Ortega y Gasset, esta situación comienza a cambiar. ${ }^{9}$ Pronto, se forman

${ }^{5}$ Cfr. San Martin, J., Phänomenologie in Spanien. Neumann, Würzburg, Konigshausen, 2005

${ }^{6}$ Dejo la ola de recepción más tardía en España aquí fuera de consideración. Hay un estudio detallado en el libro reciente de Javier San Martín, editado por San Martín, op. cit.. Debe mencionarse aquí que ha habido un renovado interés en el trabajo de Scheler entre 1910 y 1920 en España desde 1990. Véase, por ejemplo, el libro de Miguel García Baró Vida y Mundo, las investigaciones éticas de Juan Miguel Palacios en Bondad Moral e Inteligencia ética, que proceden de los primeros textos fenomenológicos, la reedición de las traducciones antiguas de El Resentimiento en la moral, Ordo amoris, Esencia y Formas de la Simpatía, (Gaos) y las traducciones de Los ídolos del autoconocimiento y de Sobre el Pudor y el sentimiento de vergüenza, por la propia autora. Un cierto renacimiento acerca de Scheler claramente se puede afirmar aquí.

${ }^{7}$ Orringer, N., Ortega y sus fuentes germánicas, Gredos, Madrid, 1979, p. 197.

${ }^{8}$ Marías, J., Ortega. Circunstancia y vocación, Revista de Occidente, Madrid, 1983, p. 69.

${ }^{9}$ Unamuno y Ortega quieren hacer que ambas filosofías sean dinámicas en España. Sin embargo, las ideas para esta reforma de la filosofía son muy diferentes para ambos. Unamuno desarrolla una filosofía existencial y se centra en personajes como Kierkegaard. Ortega, por su parte, está fuerte- 
grupos de investigadores, que son altamente receptivos a las escuelas filosóficas de otros países europeos, y se crean revistas. Ortega y Gasset juega aquí un papel decisivo y dinámico: en 1913 funda la "Liga de Educación política española”, la revista España en 1915, el El Espectador en 1916 y la conocida Revista de Occidente en 1923, que se publica ininterrumpidamente hasta el comienzo de la guerra civil en $1936 .{ }^{10}$ En este contexto, se prestó mucha atención a las escuelas filosóficas alemanas de la época y muchos se dirigieron a Alemania para estudiar filosofía y participar activamente en estas nuevas escuelas. Este fue el caso de José Ortega y Gasset, Xavier Zubiri y Manuel García Morente. ${ }^{11}$ Aunque estos autores se sintieron inicialmente atraídos por el neokantismo, su interés pronto se centró en la fenomenología, que luego se consolidó como un nuevo movimiento filosófico. Las ideas de estos fenomenólogos, especialmente Husserl y Scheler, pronto se hicieron populares en España, y la organización del movimiento fenomenológico en sus propios círculos proporcionó un patrón para que los pensadores españoles organizaran la filosofía académica en su propio país. Las estancias en Alemania de los filósofos españoles, así como los contactos con la fenomenología y los fenomenólogos de la entonces filosofía española dieron un nuevo impulso y fueron una fuente de inspiración para los pensadores españoles. Esta inspiración se sintió en dos niveles en aquel entonces. Por un lado, la fenomenología ofreció a los pensadores españoles un rico campo temático de investigación, que - como se verá en las siguientes secciones-, cada autor enriqueció con su propia tradición cultural. Por otro lado, el movimiento fenomenológico fue visto como un modelo para una reorganización de la filosofía en el propio país. En este aspecto institucional, vemos que los autores nombrados después de su regreso a España también forman escuelas fenomenológicas y organizan las actividades correspondientes. La Revista de Occidente actuó como un órgano de publicación durante esta época; contiene la mayoría de las traducciones y artículos fenomenológicos. En este sentido, se puede hablar de la apertura de una tradición filosófica, que, tanto a nivel epistémico como institucional, sigue de cerca el movimiento fenomenológico en el mundo de habla alemana

mente influenciado por una concepción racionalista de la filosofía y pasa primero por Kant y luego hacía la fenomenología. Hubo confrontaciones abiertas entre Unamuno y Ortega. Cfr. San Martin, J., Fenomenología y Cultura en Ortega. Ensayos de Interpretación, Tecnos, Madrid, 1998, p. 22.

${ }^{10}$ Lasaga Medina J., José Ortega y Gasset 1883-1955. Vida y Filosofía, Biblioteca Nueva, Madrid, 2003 , p. 63.

${ }^{11}$ A comienzos de los siglos XIX y XX, las universidades alemanas no solo eran reconocidas en España sino también en todo el mundo. Autores como el estadounidense W. James o el británico E.B. Titchener habían escrito sus disertaciones en Alemania. 
y da fuertes impulsos a la filosofía del país. Esta dinamización de la filosofía española finaliza con el comienzo de la guerra civil en julio de 1936.

En los primeros años de la dinamización de la filosofía española, las actividades fenomenológicas se organizaron principalmente en torno a dos escuelas: la Escuela de Madrid y la Escuela de Barcelona. ${ }^{12}$ La Escuela de Madrid fue diseñada por José Ortega y Gasset, Manuel García Morente, José Gaos y Ramiro Ledesma. La escuela también incluyó a Pedro Laín Entralgo (19082001), cuyo trabajo estuvo influenciado por Dilthey, Ortega, Scheler, Zubiri y Heidegger, y María Zambrano (1904-1991), quien estudió con Zubiri, García Morente y Ortega y Gasset. Después de la Guerra Civil hay una nueva edición o segunda generación de esta escuela, que incluye a Julián Marías e Hilario Rodríguez Sanz. La escuela en Barcelona incluye a Joaquim Xirau, considerado como el fundador, Eduardo Nicol y Josep Ferrater Mora. Esta escuela se disolvió después de la guerra porque sus miembros estaban en el exilio o tuvieron que enfrentarse con represiones durante la dictadura. ${ }^{13}$ Para la recepción de Scheler en ambas escuelas, las traducciones han jugado un papel importante, por lo que representan una forma importante de la recepción. Los escritores españoles se han dedicado con gran dedicación a la traducción para que el trabajo de la fenomenología sea conocido por un público más amplio en España, no solo por aquellos que entienden el idioma alemán. El español es, pues, la primera lengua extranjera en la que se han traducido muchos textos fenomenológicos. Aquí no sólo están las traducciones de los textos de Scheler, sino también los textos de los fundadores del movimiento fenomenológico como Brentano, Husserl y Pfänder, así como los textos de otros interlocutores de Scheler como Celms y Kolnai, que favorecieron la recepción de Scheler, proporcionando el marco de discusión para su filosofía. Si echamos un vistazo a las biografías intelectuales de los autores que se ocupan del trabajo de Scheler, entonces sale a la luz su importante papel como traductor de textos fenomenológicos o como generador de impulsos para tales traducciones.

\subsection{La escuela de Madrid}

José Ortega y Gasset (1883-1955), como fundador de la Escuela de Madrid, tiene innegablemente una gran importancia para la recepción de Scheler en

\footnotetext{
${ }^{12}$ Lerin J., "recepción y desarrollo de la fenomenología en España", en San Martín, J., Phänomenologie in Spanien, pp. 13-28, aquí p. 14, Mora A., "Fenomenología en el exilio I o el Colegio de Barcelona” en San Martín, op. cit., pp. 288-290, aquí p. 289.

${ }^{13}$ Perenya Blasi F., "Fenomenología en Cataluña", op. cit., pp. 295-296.
} 
España. Debido a su trabajo, es legítimamente llamado "precursor en Scheler" ${ }^{14}$ o "embajador de la fenomenología en España". ${ }^{15}$ Sin embargo, la relevancia de la figura de Ortega para la recepción de Scheler no radica en su actividad de traducción, sino en la inclusión del pensamiento de Scheler en su propio sistema filosófico y en el hecho de que da forma a toda una generación de filósofos futuros que llegan a conocer la fenomenología de sus propias manos. El pensamiento de Ortega fue moldeado por la filosofía de Unamuno, la literatura francesa de Balzac, Stendhal y Flaubert, ${ }^{16}$ y especialmente por la filosofía alemana de principios del siglo Xx. De 1905 a 1906, Ortega pasa dos semestres en Leipzig y Berlín y entra en contacto con el neokantismo. En 1907 emprendió un segundo viaje a Marburg, para estudiar la filosofía de Kant con Hermann Cohen y Paul Natorp.

Solo en su tercer viaje a Alemania, Ortega se pone en contacto con Nicolai Hartmann, Heinz Heimsoeth y Max Scheler en 1911 y se interesa mucho por el movimiento fenomenológico. ${ }^{17}$. Después de su regreso a España, Ortega comienza a profundizar su conocimiento fenomenológico con la lectura de las Investigaciones Lógicas de Husserl, para trabajar fenomenológicamente y difundir la nueva corriente filosófica en ensayos, artículos y conferencias. ${ }^{18}$ La influencia de la fenomenología en Ortega se ve reflejada ya en 1912 en tres textos: uno sobre Machado, "Los versos de Antonio Machado", uno sobre el realismo en la pintura, "Del realismo en pintura" y un tercero sobre Azorín, "Un nuevo libro de Azorín". Estos textos son importantes porque en ellos los temas relevantes son examinados con la ayuda del método fenomenológico. ${ }^{19}$

La influencia de la fenomenología, y en particular la de Husserl, es aún más pronunciada en algunas de las conferencias y artículos de 1913, que son el primer retrato de la fenomenología en España. La primera conferencia, en la que se puede ver la influencia de la fenomenología en Ortega, se llevó a cabo en el cuarto congreso de la "Asociación Española para el Progreso de

\footnotetext{
${ }^{14}$ Pintor Ramos, op. cit.

${ }^{15}$ Mulligan K., "La fenomenología realista", Ciclo de conferencias inédito en el Institut d'Estudis Catalans y la Universitat de Barcelona, Partes de ella revisadas antes del autor: Vendrell, I, "Memòria del seminari de maig de 1999", Actes i Objectes. Una anàlisi de la fenomenologia realista, Mulligan, K., (2001), en Anuari de la Societat Catalana de Filosofia, pp. 41-64.

${ }^{16}$ Lasaga Medina J., José Ortega y Gasset, op. cit., p. 26.

${ }^{17}$ Pintor Ramos A., La influencia de Scheler en el pensamiento del mundo de habla hispana en op. cit., p. 317, Lasaga Medina J., José Ortega y Gasset, op. cit., pp. 298-300

${ }^{18}$ San Martín J., Fenomenología y cultura en Ortega., p. 54 y también p. 57.

${ }^{19}$ Según San Martín, también debe notarse un cambio de actitud en los textos políticos de la época, (Cfr. op. cit., p. 54).
} 
las Ciencias" en junio de 1913. El título de la conferencia fue, "Sensación, Construcción e intuición”. En junio, julio y septiembre del mismo año, Ortega publica una serie de artículos sobre el concepto de sensación Empfindung. ${ }^{20}$ En "Sobre el concepto de sensación", Ortega presenta una reseña del libro Investigaciones sobre el concepto de la sensación, de Heinrich Hoffman. En él, Ortega habla de la fenomenología como un movimiento filosófico, que se desarrolló en Gotinga alrededor de Husserl y estuvo muy influenciado por la psicología. Ortega también traduce algunos términos fenomenológicos aquí, como la palabra experiencia como "vivencia". Esto hace que la revisión sea una referencia importante para futuras traducciones.

Sin embargo, esta grabación de la fenomenología de Husserl no es acrítica, porque Ortega criticará el giro idealista de Husserl en Ideas y de ese modo se posicionará como un fenomenólogo realista. Ya en 1914 Ortega publicó el "Ensayo de Estética a manera de prólogo". En este texto, Ortega considera imposible que el tema de investigación se convierta en un objeto y critica el idealismo de Husserl. ${ }^{21}$ La fenomenología de Ortega y Gasset es, ante todo, una fenomenología realista, como Husserl y sus alumnos lo entendieron por primera vez. Esta crítica del idealismo de Husserl afectó la comprensión de la fenomenología en España. Ortega está así cerca de los primeros fenomenólogos. Especialmente la concepción de la estética de Moritz Geiger retomada en Ortega; también de Pfänder, la imagen de lo psíquico influirá en él y especialmente las obras de Scheler sobre los sentimientos, los valores y el amor fueron bien recibidas por Ortega. En 1916 Ortega publicó el artículo en El Espectador: "Conciencia, objeto y las tres diferencias de este". El texto es un resumen de dos seminarios sobre fenomenología realizados por Ortega a lo largo de $1915 .^{22}$ Vemos aquí que Ortega ya organiza seminarios sobre temas fenomenológicos, que él mismo asume la perspectiva fenomenológica y que él entrena a los estudiantes en la tradición fenomenológica. La influencia de la fenomenología y el enfoque del pensamiento antropológico de Scheler se hacen aún más evidentes en los textos de Ortega de la década de 1920, donde muestra un interés en la antropología como una ciencia del hombre. ${ }^{23}$ Ortega publicó El tema de nuestro tiempo en 1923. En este texto, Ortega quiere

${ }^{20}$ Cfr. Ortega y Gasset J., Investigaciones Psicológicas, Revista de Occidente, Madrid, 1981, p. 219. Según San Martín, Ortega publicó en 1913 en la Revista de libros tres reseñas de la fenomenología de Husserl y su escuela, Cfr. J. Marías Acerca de Ortega, Espasa Calpe, 1991 p. 139, Madrid.

${ }^{21}$ Marías J., Acerca de Ortega, op. cit., p. 142.

${ }^{22}$ Cfr. Ortega y Gasset J., Investigaciones Psicológicas, op. cit., p. 9.

${ }^{23}$ Orringer N., Ortega, y sus fuentes germánicas, op. cit., p. 238. 
explorar al ser humano como un todo con sus diversos aspectos cognitivos, afectivos y volitivos. Ortega critica los modelos racionalistas de la razón y, por el contrario, quiere explorar otros elementos, como la afectividad. Por ejemplo, él habla de una razón vital. Aunque la influencia de Kant y Nietzsche también se puede sentir, Scheler obtiene una resonancia mucho mayor. ${ }^{24}$ El eco de las ideas de Scheler se debe notar esencialmente en dos puntos. Por un lado, está la idea de una investigación integral del ser humano en la que el elemento afectivo recibe un estatus especial. Refleja las tesis de Scheler de fases realistas en las que se entiende la afectividad como el núcleo de la persona. Por otro lado, Scheler también quería exponer la conexión de los sentimientos con la razón y, en su opinión, poner fin a la falsa oposición entre razón y sentimiento. Los sentimientos están conectados con las cogniciones porque se basan en el elemento cognitivo y porque ellos mismos tienen una función informativa: están enfocados en las cualidades especiales del mundo, los valores que de otro modo permanecerían ocultos para nosotros. Otros testimonios de esta influencia de Scheler son proporcionados por otros textos de Ortega de los años 20, donde editó el tema de la afectividad humana, los sentimientos y su conexión con los valores. Por ejemplo, en: "Vitalistmo, Alma y Espíritu" (1924), "Sobre la expresión del fenómeno cómico" (1925), "Sobre el amor en Stendhal" (1925), "Corazón y Cabeza" (1927). Este interés antropológico culminará en La rebelión de las masas.

En junio de 1928, Ortega escribe con motivo de la muerte de Scheler un ensayo para la Revista de Occidente bajo el título "Max Scheler". Cinco años después, presentará el sugerente subtítulo "Un embriagado de esencias". ${ }^{25}$ Este texto está diseñado como un elogio a Max Scheler. Es presentado como un "genio" y filósofo de la esencia y como "adán del nuevo paraíso", ${ }^{26}$ aunque, según Ortega, su obra carece de orden y sistema. También está inspirado en la imagen de Scheler en España, según la cual a Scheler le falta un poco de razonamiento sistemático y riguroso, pero ciertamente debe ser considerado como un genio filosófico. ${ }^{27}$

Los años de exilio de Ortega comenzaron en 1936: pasó tres años en Francia, tres en Argentina y tres en Portugal, regresando a España en $1945 .{ }^{28} \mathrm{El}$

\footnotetext{
${ }^{24}$ Lasaga Medina, J., José Ortega y Gasset, op. cit., p. 63.

${ }^{25}$ Ortega y Gasset J., Obras Completas, IV, Revista de Occidente - Alianza Editorial, 1947, pp. 507511, Madrid.

${ }^{26}$ Ibidem, p. 510.

${ }^{27}$ En este sentido también Pintor Ramos, A., la influencia de Scheler, p. 318.

${ }^{28}$ Lasaga Medina, J., José Ortega y Gasset, op. cit., p. 318.
} 
proyecto de renovación del país de Ortega se ve interrumpido por la guerra civil. Otros escritores de la Escuela de Madrid también contribuyeron a la recepción de Scheler a través de su trabajo como traductores y la inclusión de los pensamientos de Scheler en su trabajo. Xavier Zubiri (1898-1983) es uno de los pensadores de la Escuela de Madrid. Aunque Zubiri estudia con Ortega y Gasset, aprende del movimiento fenomenológico de Marcelino Arnaiz y Leon Nöel, dónde escribió su tesis sobre "El problema de la objetividad en Husserl". ${ }^{29}$ En Madrid, luego hace su doctorado con una disertación sobre la fenomenología del juicio. El pensamiento de Zubiri solo se ve influenciado secundariamente por Scheler, porque se basa principalmente en Husserl y Heidegger, pero en 1935 hizo una gran contribución a la recepción de Scheler con la traducción de Muerte y Supervivencia y Ordo amoris.

Manuel García Morente (1886-1942) estudió en Francia la filosofía de Bergson y en Alemania la filosofía de los neokantianos. En 1912 obtuvo una cátedra en el Departamento de Ética de la Universidad de Madrid y allí, bajo la influencia de Ortega y Gasset, volcó su interés por la fenomenología. Su trabajo consiste en una amplia actividad de traducción. Él transcribió escritos de Kant, Spengler y Rickert, pero especialmente los trabajos de autores fenomenológicos. En 1929 aparece la traducción de Sobre el origen del conocimiento moral de Brentano y las Investigaciones Lógicas de Husserl, esta última traducida por García Morente junto con José Gaos. Un año después, apareció la traducción de David Katz de El mundo de las sensaciones táctiles y, en 1931, apareció la Fenomenología de la voluntad de Pfänder. Durante la Guerra Civil García Morente emigró a París y luego a Argentina, donde obtuvo su propia cátedra. En 1938 regresa a España, se convierte en sacerdote católico y recurre a Tomismo. La influencia de Scheler es evidente en los escritos éticos de Morente. Especialmente en el ensayo de Morente "Juicios de Valor" (1918) y en sus "Ensayos sobre el progreso" (1932), y el ciclo de conferencias dadas en Argentina "El ideal y el estilo" (1938) fue el desarrollo de un concepto de valor en la producción de Scheler.

También José Gaos (1900-1969), otro miembro de la Escuela de Madrid, se orientó inicialmente hacia el neokantismo. En 1921, Gaos va a Madrid, asiste a los seminarios de García Morente y Zubiri y se mueve en el círculo de estudiantes de Ortega. Bajo esta influencia, recurre a la fenomenología y lee

${ }^{29}$ Conill J., "La Fenomenología en Zubir", en San Martín, Fenomenología en España., supra, aquí p. 44. 
a Husserl, Hartmann, Scheler, Dilthey y Heidegger. ${ }^{30}$ Durante la guerra civil emigró a México en 1938 y encontró un puesto en la UNAM. La contribución de Gaos a la recepción de Scheler no se detiene en su enseñanza y conferencias dadas en España y América Latina, sino también en su trabajo de traducción intensiva. Gaos ha traducido al español a Fichte, Dilthey, Brentano, Husserl, Celms y Scheler. Como ya se mencionó, tradujo junto con García Morente las Investigaciones Lógicas de Husserl y las cuatro primeras Meditaciones Cartesianas. ${ }^{31}$ Sobre Brentano, tradujo partes de Psicología desde un punto de vista empírico. Sin embargo, Scheler es el autor al que Gaos dedica la mayor parte de su trabajo de traducción. Así, en 1927 aparece $E l$ resentimiento en la moral, en 1929 El puesto del hombre en el cosmos, en 1935 Sociología del saber y en1943 Esencia y formas de la simpatía. Entre sus numerosas publicaciones fenomenológicas se encuentran las siguientes: La crítica del psicologismo en Husserl (1928), Dos exclusivas del hombre: la mano y el tiempo (1945), Ensayos sobre Ortega y Gasset (1957), Introducción a la fenomenología (1960). Por último, se nombrará a otro estudiante de Ortega, a saber, Ramiro Ledesma (1905-1936). Ledesma estaba muy influenciado por el pensamiento de Husserl, Scheler y Heidegger. Es digno de mención el artículo "La última incógnita de M. Scheler" del año 1929. Ledesma fue asesinado durante la guerra civil.

El destino de los autores de la Escuela de Madrid también se vio afectado por la guerra civil. Su propia producción filosófica sufrió pérdidas humanas y culturales, y la actividad de traducción activa se interrumpió hasta la década de $1940 .{ }^{32}$

\subsection{La escuela de Barcelona}

En cuanto a la escuela de Barcelona, Joaquín Xirau (1885-1946) hizo una contribución importante a la recepción de Scheler, no a través de las traducciones, sino organizando las actividades de la escuela e incorporando el pensamiento

\footnotetext{
${ }^{30}$ Lasaga Medina, J., La presencia de Husserl en Ortega, pp. 29-42.

${ }^{31}$ Esta traducción se basó en un manuscrito dado por Husserl Ortega y Gasset en 1934. Dado que el manuscrito se perdió en la Guerra Civil, la traducción de 1942 solo podía contener las primeras cuatro meditaciones. Cfr. Lasaga Medina, J., La presencia de Husserl en Ortega, p.30.

${ }^{32}$ La segunda generación de la Escuela de Madrid también tiene numerosas traducciones para agradecer. Julián Marías 1914-2005, que estudió en Madrid en Ortega y Gasset, Zubiri, Gaos y García Morente, tradujo 1940 De lo eterno en el hombre. Hilario Rodríguez Sanz, alumno de García Morente, es el autor de la primera traducción a un idioma extranjero del libro de formalismo de Scheler en 1942. Rodríguez Sanz recibió su doctorado en 1945 en Madrid sobre la filosofía de la persona de Scheler.
} 
de Husserl y Scheler en su propia la filosofía. Xirau estudió en Barcelona y París y tiene un doctorado en Madrid, donde asistió a varios seminarios de Ortega y Gasset. En Barcelona organizó un coloquio filosófico, donde Zubiri, García Morente, Gaos, Paul Ludwig Landsberg, Charlotte Bühler y Jean Piaget impartieron seminarios. Los seminarios de Landsberg versaban sobre San Agustín, Nietzsche y Scheler. En 1939, Xirau emigra a México, donde dirige el Departamento de Metafísica de la UNAM. Sus obras de orientación fenomenológica son La filosofía de Husserl (1941) y La fenomenología de los valores en relación con la ética y el derecho (1929), donde se puede sentir la influencia de la antropología de Scheler. Cabe destacar el L'amor i la percepció dels valors (1936), publicado en catalán, y la obra Amor y Mundo (1940) en español. En estos escritos, Xirau recoge y desarrolla el postulado antropológico del amor de Scheler como el núcleo de la personalidad. En resumen, podemos decir que hubo una recepción intensiva de la antropología filosófica de Scheler en España. Esta recepción se organizó en torno a la escuela de Madrid y la escuela de Barcelona. Los protagonistas de estas escuelas han contribuido a la introducción del pensamiento de Scheler en España a través de un gran número de traducciones y la organización de actividades fenomenológicas.

\section{Valor, sentimiento de valor y sentimiento}

\subsection{Scheler: el hombre como un ser sensible y la objetividad de los valores}

Si consideramos la inclusión y el desarrollo del pensamiento de Scheler entre los autores españoles, podemos descubrir que su enfoque se centra en el tema de la afectividad humana. ¿Cuál de las tesis de Scheler fue particularmente popular en España? En el curso de su investigación sobre ética, Max Scheler desarrolla su propia teoría de valores, que se refleja principalmente en el libro de Formalismo. La teoría del valor de Scheler establece que los valores tienen su propia realidad independiente del ser humano y que solo son accesibles para nosotros a través de ciertos actos de "sentir". La posición de Scheler se llama realismo de valores y contrasta fuertemente con el emocionalismo, que entiende los valores como proyecciones de nuestros estados emocionales en el mundo, según los cuales el paisaje es "triste", porque siento luto al ver el paisaje y luego transfiero esto a ese paisaje. El realismo de valores también contrasta con el disposicionalismo, que percibe los valores como 
disposiciones. Continuando con el ejemplo anterior: según esto, ese paisaje sería "triste" porque tiene la disposición de inducir este sentimiento en las personas.

Los valores también existen según Scheler en una determinada clasificación de objetivos y también se presentan en un sistema polar. Los valores sensibles se dividen en agradables y desagradables, los valores vitales en noble e innoble, los valores espirituales en las parejas correctas e incorrectas (para la ética), bellas y feas (para la estética) y verdaderas y falsas (para el conocimiento). Por último, están los valores religiosos de lo santo y lo profano. ${ }^{33} \mathrm{La}$ ontología de valores de Scheler también requiere una epistemología particular: ¿Cómo son accesibles los valores? ¿Cómo sabemos que algo es elegante, malo, repugnante, peligroso, etc.? La respuesta de Scheler es radical en este punto: Los valores son accesibles para nosotros gracias al sentir. Solo porque tenemos la capacidad de sentir, también podemos comprender. Sin esta habilidad, se nos daría el mundo sin cualidades. El sentimiento es responsable del reconocimiento de los valores y la acción motivadora correspondiente. Para la ética, esto significa que lo que uno debe hacer no está determinado por deberes o normas (como es el caso con la ética formal de Kant), sino que se descubre a través de la función afectiva del sentimiento. En este contexto de una ética de valores, el sentir intencional (Fühlen) y los sentimientos no intencionales (Gefühlen) son para Scheler (pero también de otros fenomenólogos como Geiger, Stein y Kolnai) de gran importancia. El sentir intencional (Fühlen) a menudo se describe como un "órgano de adquisición de valor" o "toma de valor". ${ }^{34}$ Gracias a él, el mundo se presenta, no como un nivel monótono y neutral, sino como un horizonte con ciertas cualidades en las que podemos orientarnos. Aquí Scheler formula un postulado antropológico muy fuerte, según el cual el sentimiento (es decir, una función afectiva) es más fundamental que cualquier acto de pensar o querer. ${ }^{35}$

Esto plantea la pregunta de si este sentir intencional es al mismo tiempo un sentimiento o si forma una categoría separada. En el papel del percibir sentimental, las mentes difieren en fenomenología. Mientras que, para Husserl, Stein y Kolnai el percibir intencional de valor y los sentimientos son

${ }^{33}$ Cfr. Henckmann, W., Max Scheler, C.H. Beck, Múnich 1998, p. 106.

${ }^{34}$ Scheler, M., El formalismo en la ética y la ética del valor material, en GW II, p. 269, Para que este sentimiento de valores no parezca irracional, Scheler se une a la tesis de Husserl, según la cual, además de la percepción, la apreciación también es posible. Husserl explica la toma de valor en sus "Conferencias sobre ética y teoría del valor", Hua XXVIII, ver Henckmann, W., op. cit.

${ }^{35}$ Scheler, Ordo Amoris. 
sinónimos (es decir, la repugnancia es lo mismo que sentir lo repugnante), para Scheler, Geiger y Ortega y Gasset, el sentir está severamente separado de los sentimientos ${ }^{36}$ Para los últimos autores, la percepción sentimental captura los valores, y los sentimientos son respuestas a los valores captados en el percibir sentimental. Para seguir con el ejemplo anterior: lo desagradable se detecta en él, y cuando se trata de una respuesta razonable, sentimos disgusto. Por lo tanto, esta tesis no concibe los sentimientos como actos de cognición y postula la existencia de una facultad humana que no puede describirse como meramente cognitiva en el sentido tradicional de la palabra ni meramente afectiva y que nos transmite valores. El sentir es, por lo tanto, un tipo de facultad psíquica que puede describirse como cognitivo-afectivo. Con el postulado del sentimiento como un medio para conocer los valores, Scheler ya muestra cuán débiles son los límites entre los actos que la tradición ha caracterizado como cognitivos y que se caracterizan por el conocimiento, y aquellos actos que fueron caracterizados como afectivos debido a su potencial para inducir impulsos físicos en nosotros.

El sentir está relacionado con los sentimientos. Hay cuatro tipos principales de sentimientos asociados con los valores percibidos: Sentimientos sensibles, sentimientos vitales, sentimientos emocionales y sentimientos religiosos, según los valores registrados. En la función de sentirse a sí mismo, es importante enfatizar que Scheler lo entiende como el núcleo de la persona. En el texto Ordo Amoris desafortunadamente ignorado por la fenomenología actual, Scheler presenta una tesis que retomará y trabajará la filosofía española. Especialmente lo encontramos en Ortega, Morente y Xirau nuevamente. Se entiende como un postulado antropológico que el hombre es un ser sensible. Sin embargo, cada persona tiene que sentir de cierta manera, lo que constituye el núcleo de su persona. Sentirse de esta manera está determinado por el contexto histórico, social y cultural y no solo por factores individuales, pero la idea es que es diferente para cada persona, por lo que es único. El ordo amoris, que es inherente a cada ser humano, marca las líneas básicas de su personalidad y también determina su forma de abrir valores: algunas personas

${ }^{36}$ Mulligan, K., Husserl on the "Logic" of Valuing, Values and Norms, en Centi, B \& Gigliotti, G. ed. Fenomenologia della Ragion Practica. L'Etica di Edmund Husserl, Bibliopolis, Nápoles, 2004, pp. 177-225; también Feelings, preferences and Values, Unveröffend, Vortrag, Lisboa y Neuchatel, 2005; igualmente Schelers H., "Was man alles fühlen kann. Wie vielerlei man fühlen kann", en H. Landweer \& U. Renz, ed. Klassische Emotionstheorien von Plato bis Wittgenstein, de Gruyter, Berlín, 2008, Vendrell Ferran, I., Die Emotionen Gefühle in der realitischen Phänomenologie. Akademie, Berlín, 2008. 
prestarán atención a algunos valores y pasarán por alto a los demás; cada uno muestra un sistema de preferencias diferente.

\subsection{Ortega y Gasset: la ciencia de los valores y el valor de la vida}

Ortega tiene en su trabajo dos concepciones diferentes del concepto de valor: fluctúa entre una concepción realista de valores y una disposición de valores. La concepción de valor-realista de los valores está fuertemente influenciada por la ontología y la epistemología de valores de Scheler. En 1923, Ortega y Gasset escribió el ensayo ¿Qué son los valores?, que sigue siendo el único texto sobre el tema de los valores en el mundo de habla hispana, hasta la aparición de la traducción del libro de Scheler, El formalismo. En el referido ensayo, Ortega y Gasset representa una posición de valor realista, según la cual se pueden percibir valores como otros objetos. Él está así explícitamente en la línea de Husserl y Scheler. Los valores no se remontan a lo agradable o deseado, ni se derivan de las normas, ya que según Ortega son "objetivos" y poseen su propia naturaleza. ${ }^{37}$ Los valores se caracterizan por su materia, calidad y rango.

¿Qué tipo de "objetividad" ahora viene a los valores? Ortega y Gasset afirma que los valores son cualidades "irreales" y que tienen una objetividad "virtual" porque son "objetivos" pero no reales como los árboles u otras personas. La objetividad de los valores es como la de colores o números. Los valores existen en orden de clasificación y se pueden clasificar como positivos o negativos. Respecto a la posibilidad de capturar los valores, Ortega y Gasset afirma que estos se vuelven accesibles para nosotros en un "sentimiento"; Él está de acuerdo con la opinión de Scheler. Según Ortega, "sentir" es una "función cognitiva" y funciona como ver o entender. Para explicar esto, trabaja con un paralelismo entre el "sentimiento" (de los valores) y la percepción (de las cosas). Sin embargo, según Ortega, "sentir" y "percibir" difieren en varios aspectos. Por un lado, ambos tipos de información a menudo no se ejecutan en paralelo. Podemos percibir una cosa y sus valores todavía pueden ser inclinados sobre nosotros. Por el contrario, podemos captar valores sin darnos una cosa con claridad. Este es el caso, por ejemplo, cuando uno "siente" el mundo de la justicia perfecta, sin saber en qué situación real esta justicia se podría realizar. ${ }^{38}$ Por otro lado, la percepción y el sentimiento son de naturaleza diferente: el primero siempre tiene lugar a través de som-

${ }^{37}$ Ortega y Gasset J., Obras completas, vol. VI, op. cit., pp. 325-328.

${ }^{38}$ Ibidem, p. 331. 
bras y a través de percepciones sucesivas de lados (de una cosa), el segundo es siempre "transparente" o "absoluto y matemático", 39 es decir directamente para Ortega. Aquí él establece una comparación entre la ciencia de los valores, que él llama "estimativa" y las matemáticas. Ortega termina el ensayo con dos comentarios. Según Ortega, la moral y los valores de lo útil, a diferencia de otros tipos de valores, están condicionados por la existencia de su objeto. Esto significa que, por ejemplo, el "bien moral" solo se puede atribuir a las personas, mientras que el valor de lo "bello" puede atribuirse a diferentes tipos de objetos. La otra observación se refiere a la clasificación de los valores, que recuerda a la propia clasificación de Scheler. Ortega y Gasset distinguen entre valores de utilidad y valores vitales. Así encontramos cómo Ortega y Gasset establece la tesis de la separación entre sentimientos y sentimientos y proporciona argumentos adicionales en esta dirección.

En El tema de nuestro tiempo de 1923, Ortega presenta una visión diferente del concepto de valor. ${ }^{40}$ En este texto, los valores están relacionados con la vida, porque aquí está la realidad vital, que tiene una función fundamental para los valores. La realidad de los valores se basa así en la realidad de la vida. ${ }^{41}$ Con esta visión, Ortega se aleja del valor-realista de los valores de dos maneras. La clasificación de los valores postulados aquí es diferente de la clasificación de Scheler y también difiere de la clasificación de Ortega en Qué son los valores. Allí el valor más elevado era lo sagrado, aquí es la vida. De hecho, los fenómenos espirituales en sí mismos se basan en la vida, incluso si luego se nos presentan con cierta objetividad y aparecen como si fueran entidades independientes. ${ }^{42}$ Además, como los valores dependen de la vida humana, su trascendencia se pone en tela de juicio. ${ }^{43}$ Es el hombre mismo el que hace los valores. Porque Ortega, entiende los valores como posibilidades que los humanos tienen que poner en orden de prioridad, dependiendo de cómo se ve realmente su proyecto vital. Por lo tanto, los valores no tienen medios de subsistencia independientes del hombre, pero son posibilidades para las cuales la vida decide y que también se hacen realidad dependiendo

\footnotetext{
${ }^{39}$ Ibidem.

${ }^{40}$ Mateo, M. S., "Los Valores y Max Scheler y Ortega", en Humanitas. Revista de la Facultad de Filosofía y Letras, Universidad Nacional de Tucumán, vol. IX, 1961, pp. 157-170, aquí p. 164.

${ }^{41}$ Ortega y Gasset, J., Tema de nuestro tiempo, en Obras Completas, vol. III, op. cit., p. 596.

${ }^{42}$ Mateo, M. S., op. cit., p. 166.

${ }^{43}$ Ortega y Gasset, J., Obras Completas, vol. III, op. cit., pp. 601, 604.
} 
de la edad histórica respectiva. ${ }^{44}$ Este punto de vista de Ortega lo acerca más al disposicionalismo.

\subsection{García Morente: Valor y progreso}

La obra filosófica de Manuel García Morente ha recibido poca atención hasta ahora y fue oscurecida por su extenso trabajo como traductor. Me gustaría anotar aquí algunas ideas de Morente. En 1918, Morente publicó un ensayo titulado Los juicios de valor en dónde pregunta cuáles son los valores. Morente busca la respuesta, no en Kant y sus estándares éticos, que él conoce bien de sus traducciones, sino decididamente en la fenomenología de aquel momento con referencia directa a Scheler, Hildebrand, Hartmann y Ortega y Gasset, ${ }^{45}$ cuyo ensayo sobre valores antes mencionado se publicaría cinco años después. En 1932, las primeras ideas de Morente sobre los valores ya se reflejaron en el discurso pronunciado en la Academia de Ciencias Morales y Políticas en Madrid, publicado en el libro Ensayo sobre el progreso. Las principales preguntas de Morente son: ¿cuáles son los valores? y ¿cómo se puede hablar de progreso?

Morente representa una posición de valor realista en la línea de Scheler, según la cual los valores son independientes tanto del comportamiento humano como de la existencia de los objetos que se nos otorgan. Morente entiende los valores como "propiedades irreales". ${ }^{46}$ En su ontología hay tres clases de objetos: las cosas reales son objetos ideales y valores irreales. ${ }^{47}$ Por lo tanto, los valores no tienen existencia, pero son válidos y tienen valor: "Los valores no son, no tienen, sino que precisamente valen, tienen valor". ${ }^{48}$ Según Morente, los valores se caracterizan por tres características: materia, polaridad y jerarquía. Para representar la posición realista del valor, Morente desarrolla tres argumentos contra el emocionalismo, que pueden denominarse: argumento de contradicción, argumento temporal y argumento de la fuerza. El argumento de contradicción establece que los valores no son proyecciones de nuestros sentimientos, ya que a menudo encontramos algo valioso incluso si no nos gusta. Que algo puede ser percibido como valioso, sin embargo, no provoca un sentimiento positivo en nosotros. El argumento temporal habla en contra del emotivismo, porque a menudo el sentimiento correspondiente

\footnotetext{
${ }^{44}$ Ibidem.

${ }^{45}$ Palacios, J. M., Prólogo, en García Morente M., Ensayos sobre el progreso, Madrid, 2002, p. 10.

${ }^{46}$ García Morente, M., Ensayos sobre el progreso, Encuentro, Madrid, 2002, p. 46.

${ }^{47}$ Ibidem, p. 51.

${ }^{48}$ Ibidem, p. 62.
} 
acaece después de la detección de un valor y no antes, de modo que aquí no es posible ninguna proyección. El argumento de la fuerza dice que la proyección a menudo no es lo suficientemente fuerte como para motivarnos y guiarnos. ${ }^{49}$ Con este tour de force contra el emocionalismo, Morente desarrolla su propia posición de valor-realista, según la cual los valores se entienden como preferencias. ${ }^{50}$ Los valores son disposiciones del mundo para darnos una preferencia. Esta posición distingue a Morente de las tesis de Scheler y lo acerca al disposicionalismo.

Morente entiende el progreso como la realización de valores a través del esfuerzo humano. ${ }^{51}$ Aquí la capacidad humana de captar valores juega un papel importante. Porque sólo gracias al sentimiento los valores son accesibles para nosotros; aquí Morente retoma las ideas de Scheler. En 1938, durante una gira de conferencias en Argentina, Morente intenta especificar la capacidad humana del sentir. Morente habla del estilo que cada uno de nosotros tiene cuando percibimos los valores. ${ }^{52}$ Son sus propias preferencias de "corazón" las que expresan la personalidad íntima del hombre y constituyen el estilo personal. En estas palabras, en mi opinión, se puede encontrar la idea de Ordo Amoris de Scheler; Zubiri había traducido este texto al español en 1934. En sus últimos años, bajo la influencia del tomismo, Morente quiso revisar su concepto de valor de 1932. De este experimento sólo quedaron fragmentos debido a la muerte de Morente. ${ }^{53}$

\section{El amor y su significado antropológico}

\subsection{El amor en la fenomenología de habla alemana}

Como parte del proyecto fenomenológico temprano de una ética de los valores, el tratamiento de los sentimientos en Brentano y sus discípulos recibió gran atención. Los primeros fenomenólogos asumieron que los humanos tienen la habilidad de reconocer lo que es valioso y actuar en consecuencia. Esta habilidad es de naturaleza afectiva, y el sentimiento juega un papel central en la vida humana. No es de extrañar entonces que las emociones y otros fenómenos afectivos (un lugar prominente del amor) hayan recibido atención especial. Particularmente en las obras de Pfänder y Scheler encontramos ela-

\footnotetext{
${ }^{49}$ Ibidem, p. 48.

${ }^{50}$ Ibidem, p. 49

${ }^{51}$ Ibidem, p. 57.

${ }^{52}$ Citado después por Palacios, J.M., en el prólogo,op. cit., p. 15 a.

${ }^{53}$ Ibidem, p. 17.
} 
boradas concepciones del amor y su posición especial dentro de los fenómenos afectivos.

Es sorprendente que el amor en general no se conciba como un sentimiento. Pfänder, por ejemplo, entiende el amor en su Psicología de las disposiciones de ánimo como disposición de ánimo (Gesinnungen) y, por lo tanto, como fenómenos diferentes de los sentimientos. Como disposiciones afectivas, el amor y el odio no fluctúan entre los polos del placer y el dolor, lo que significa que el amor doloroso y el odio lujurioso son posibles. Voigtländer y Stein también enfatizan la peculiaridad del amor al referirse a la conexión con la sexualidad y al designar el amor como una disposición afectiva. ${ }^{54}$

Scheler ve el amor como algo fundado en cualquier otro acto del sentimiento, pensamiento y voluntad. Según Scheler, este es el núcleo de nuestra personalidad y determina nuestra vinculación con el mundo. ¿Cuál es la diferencia entre el amor y los sentimientos? Los sentimientos son respuestas a los valores percibidos, pero el amor, según Scheler, no lo es. Cuando amamos, no respondemos a un valor percibido, porque amamos a personas con déficits de valor y, por otro lado, podemos odiar a personas valiosas. En consecuencia, el amor y el odio no se pueden equiparar con un sentido de valores o con reacciones a los valores. Según Scheler, el amor y el odio son los responsables de descubrir algunos valores y mantener a los demás inclinados. En la medida en que son responsables de hacer que el mundo sea accesible para nosotros de cierta manera, forman el núcleo de nuestra personalidad. El amor y el odio se entienden como movimientos. En su caracterización positiva, el amor es ser un movimiento en la dirección de un mayor valor posible sin dar valor. El odio es el movimiento en la dirección del valor inferior. ${ }^{55}$ El movimiento no tiene que ser entendido en el sentido de un comportamiento de aspiración. Más bien, es un movimiento que pretende ser más alto en el valor del objeto amado, lo que permite su descubrimiento. En este sentido, el amor no busca nuevos valores en el objeto, ya que esto sería un signo de falta de amor. Amamos un objeto tal como es y con todos sus valores y no como debería ser. Debido a su función, el amor y el odio tienen un lugar especial en la teoría ética y antropológica de Scheler.

\footnotetext{
${ }^{54}$ Voigtländer, E., "Comentarios sobre la Psicología de los Conceptos”, en Neue Munich Philosophical, Heller, Hg. E. y Löw, Leipzig,1933, pp. 143-164, Stein, E., Sobre el problema de la empatía, Hall, 1917.

${ }^{55}$ Scheler, M., Esencia y formas de simpatía, en GW VII, pp. 9-258, aquí p. 159, también p. 191.
} 
Como el amor nos hace humanos, determina nuestro acceso al mundo y permite el descubrimiento de valores. Esto le da al amor un lugar especial tanto en la vida del individuo como en la historia, porque gracias al amor, tanto a los seres humanos como a la historia, porque gracias al amor, el hombre y la humanidad pueden desarrollarse aún más. El tema del odio es secundario a la visión del amor de Scheler como un fenómeno mucho más amplio y más amplio que el odio. El odio, sin embargo, siempre se considera un modo deficiente. Sin embargo, Scheler trata el odio en un ensayo de 1912 acerca del resentimiento, donde ve el resentimiento como una forma de odio.

La posición especial de amor en la fase realista de Scheler también influirá en la filosofía española. Las ideas de Scheler sobre el amor son retomadas, desarrolladas, criticadas y dan lugar a reflexiones independientes de gran originalidad.

\subsection{Filósofos españoles y su concepción del amor}

\subsubsection{Ortega y Gasset: pseudo amor y amor auténtico}

Los estudios sobre la recepción de la fenomenología en España a menudo han considerado que la influencia de la concepción de Scheler de los sentimientos y el amor es insignificante para la filosofía española. Sin embargo, una mirada a la filosofía de Ortega muestra que el amor es el hilo conductor de su trabajo. La concepción del amor de Ortega está influenciada por varias tradiciones y autores: para mencionar algunos tenemos a la literatura francesa, Unamuno y la fenomenología de Pfänder y Scheler. De Stendhal, Ortega descubre que hay amor que no es amor. El amor es un fenómeno que no escapa al poder del engaño. Unamuno influye en la idea de Ortega de que el hombre es un ser sensible. En contraste con Unamuno, que caracteriza la naturaleza humana a través de una afectividad negativa y trágica, Ortega coloca el amor en el centro de la persona. Ortega también se hace cargo de la concepción de los sentimientos de Pfänder como fenómenos de calidez emocional y la idea de Scheler de Ordo Amoris, la posibilidad del amor ilusorio y la idea de que el amor es un movimiento de menor a mayor valor. El interés de Ortega por el amor, a diferencia de los fenomenólogos realistas, no está motivado por un proyecto ético, sino por uno antropológico. Ya en 1914, en las Meditaciones del Quijote, Ortega entiende la filosofía como una ciencia del amor en el sentido del amor intellectualis de Spinoza. El amor nos conecta con el mundo, 
expande nuestra individualidad y hace que el valor de las cosas sea visible para nosotros. ${ }^{56}$ La idea es que, gracias al amor, podemos entender el mundo.

En el trabajo de Ortega hay un interesante artículo sobre la posibilidad del engaño. En El amor en Stendhal, Ortega se dedica a una nueva investigación de este fenómeno. Comienza con una crítica de la teoría de la cristalización del amor de Stendhal. La teoría de Stendhal en De L'amour establece que el amor es un proceso que surge en varios pasos, tales como: 1. La admiración, 2. La celebración del placer, 3. La esperanza, 4. El despertar del amor, 5. La primera cristalización en la que el amante piensa en las perfecciones del amado y piensa que también es amado, 6. Surgimiento de la duda, 7. La segunda cristalización, que es volver a la convicción de que uno es amado. Estos pasos sirven para hacer que la criatura amada sea siempre nueva. Este procedimiento debería ser similar al proceso de cristalización en las minas de sal de Salzburgo, de ahí el término "cristalización" para el surgimiento del amor. Según Ortega, esta teoría es errónea, porque en ella el amor será descrito como algo "hecho" por el amante con la ayuda de la imaginación. Por lo tanto, es necesario que el sujeto atribuya propiedades al objeto, de modo que básicamente se debe hablar de una ilusión porque el objeto puede no poseer estas propiedades. Según Ortega, esto no es amor, sino "pseudo amor". Porque el amor debe descubrir las propiedades del objeto y no solo atribuirlo al objeto, si el objeto realmente no tiene estas propiedades. La descripción de Ortega y Gasset del pseudo-amor se asemeja a la descripción de los llamados "sentimientos aparentes" (Scheingefühle) en el trabajo de otros fenomenólogos. Por ejemplo, Willy Hass y Alexander Pfänder hablan en general de "sentimientos presentados", Scheler de "ilusiones emocionales", "sentimientos ficticios" o "fantasías emocionales". ${ }^{57}$ Con estas expresiones, los autores nombrados quieren describir el caso en que nos atribuimos a una emoción sin tenerla. En estos casos, y a través de la imaginación, incluso sentimos la emoción imaginada. ${ }^{58}$ Max Scheler examina esto en el caso de las ilusiones de vergüenza y las fantasías de remordimiento y Else Voigtländer específicamente en el caso del "amor ilusorio o fantástico". En este sentido, podemos ver que el artículo de Ortega y Gasset sigue claramente el proyecto de los primeros fenomenólogos para proporcionar un análisis de los sentimientos. En "Sobre

\footnotetext{
${ }^{56}$ Marías J., Ortega. Circunstancia y vocación, Revista de Occidente, Madrid, 1983, p. 342.

${ }^{57}$ Scheler, M., Idealismo-Realismo, en GW IX, pp. 183-242, aquí p. 213.

${ }^{58}$ Scheler, M., De lo eterno en el hombre: arrepentimiento y nuevo nacimiento, en GW V. Véase también del mismo autor, Sobre el pudor y el sentimiento de vergüenza, en GW X, p. 100.
} 
la cultura" (1917), "Sobre la historia del amor" (1926) y "Estudios sobre el amor" (1941), Ortega y Gasset retoma la tesis de Scheler de que el amor y el odio no son respuestas a los valores, como también las tesis de Pfänder que el amor y el odio poseen "temperatura anímica", que significan unión o desvinculación del objeto, y que son actos centrífugos en curso. ${ }^{59}$ Sobre la base de estas tesis, él hace su propia contribución caracterizando el amor y el odio como una "acción interna" 60 hacia su objeto: "El amor y el odio, sin embargo, son una acción constante, ya sea cerca o lejos, el amor envuelve a su sujeto en una atmósfera propicia, esté es caricia, alabanza, afirmación. El odio envuelve al suyo, con no menos fuego, en una atmósfera desfavorable; se lo come, lo deshidrata como un siroco brillante, prácticamente lo levanta y lo destruye. No es necesario, para decirlo nuevamente, que esto realmente suceda. Hablo de la intención que reside en el odio, de la acción interna que hace que el odio odie". ${ }^{61}$ Cabe destacar que Ortega trató el amor y el odio, a diferencia de Scheler, como "sentimientos", aunque también les proporciona una posición especial dentro de esta clase. El amor y el odio son "sentimientos activos" y, como tales, son diferentes de los "sentimientos pasivos" de alegría y tristeza. ${ }^{62} \mathrm{Si}$ bien estas últimas "reacciones de respuesta" son valiosas, los sentimientos activos de amor y odio hacen algo con el objeto al que apuntan, revelando algo de nuestra personalidad.

El enamoramiento es también el tema del análisis de Ortega y Gasset. Su tesis principal es que el "enamoramiento" es un fenómeno de atención. ${ }^{63}$ Enamorarse significa para Ortega que se ha captado la atención de un objeto. Como resultado, el mundo desaparece y el objeto de nuestra atención se vuelve más importante debido a la pérdida de otro posible punto de comparación. Sin esta reducción del mundo, que tiene algo de hipnotismo y éxtasis, no podemos enamorarnos, según Ortega. Por cierto, es importante tener en cuenta que, según Ortega, este aprisionamiento de la atención no solo es posible en el amor, sino también en el odio. Incluso habla de la posibilidad de "odiarse a uno mismo" para caracterizar el fenómeno en el cual, un hombre, a pesar de

\footnotetext{
${ }^{59}$ Ortega y Gasset, J., "Sobre el amor”, it Meditaciones, Deutsche Verlag-Anstalt, Stuttgart, 1957, p. 97.

${ }^{60}$ Ibidem, p. 104.

${ }^{61}$ Ibidem, en el mismo sentido esta "intención de destrucción" del odio como un aspecto constitutivo de la emoción de Kolnai, cfr., Kolnai A., Versuch über den Hass, en Philosophisches Jahrbuch, 48, 1935, pp. 147-187.

${ }^{62}$ Ortega y Gasset J., Sobre el amor, op. cit., p. 105, también p. 129.

${ }^{63}$ Ibidem, p. 131.
} 
odiar a otro, muestra interés en él y organiza su mundo alrededor del objeto odiado. En el caso del amor, la reducción de la atención no es negativa, ya que esta exclusividad de la atención enfatiza en el objeto cualidades importantes que de otro modo habrían permanecido en la oscuridad. En el amor, así Ortega sigue a Scheler, se descubren nuevos valores en la persona amada. Al igual que Scheler, Ortega afirma que este tipo de atención caracteriza mejor a una persona: varía de persona a persona, revelando la dirección de sus intereses y la forma en que el hombre se encuentra con el mundo y con los demás. Así, la elección del amor revela el verdadero carácter de una persona: "El tipo de humano que preferimos en el otro ser caracteriza la naturaleza de nuestro propio corazón" ${ }^{64}$ Aquí, también, el amor se entiende como una actitud fundamental, que se considera el núcleo de la persona y determina el acceso al mundo: "Entonces el amor es por su propia elección natural. Y a medida que se eleva desde el núcleo de la persona, desde las profundidades del alma, los principios de selección que los determinan son al mismo tiempo las valoraciones más íntimas y secretas que dan forma a nuestro carácter individual". ${ }^{65}$ En este sentido, el amor de los amantes es una elección desde lo más profundo de nuestros corazones y esto tiene la consecuencia contradictoria de que la elección del amor no es tan libre como se piensa, sino que depende del carácter básico del individuo. ${ }^{66} \mathrm{El}$ tema de la elección del amor es muy importante en Ortega, combina esta idea con la idea de Ordo Amoris en Scheler. Él entiende el Ordo Amoris como "ratio essendi" y "ratio cognoscendi", es decir, como lo que caracteriza nuestra personalidad y determina nuestro acceso al mundo. Ortega incluso afirma que el Ordo Amoris de cada persona se revela más claramente en la elección del amor. ${ }^{67}$ También en "Para una psicología del hombre interesante" (1925) hay más explicaciones sobre el amor. Según Ortega, cuando amamos a alguien, podemos intuir y apreciar la naturaleza del otro. Esto nos hace atentos y curiosos al otro. ${ }^{68}$

El interés de Ortega en el tema del amor surge muy temprano en su trabajo y es influenciado repetidamente por las tesis de Scheler a lo largo de los años. Ambos autores entienden la comunidad humana del amor como característica

${ }^{64}$ Ibidem, p. 169.

${ }^{65}$ Ibidem, p. 177.

${ }^{66}$ En los textos de María Zambrano sobre el amor también se pueden encontrar algunas ideas de Scheler, los cuales esta autora probablemente ha asumido implícitamente por el trabajo de Ortega y Gasset.

${ }^{67}$ Ver Mateo, M. S., "Los valores en Max Scheler", op. cit., p. 163.

${ }^{68}$ Ortega y Gasset, J., Obras completas, vol. IV, op. cit., p. 478. 
de la existencia humana. Además, ambos autores se vuelven contra la tesis de Kant de que el amor y los sentimientos en general no son parte de la ética. ${ }^{69}$ A pesar de la influencia de Scheler en Ortega y a pesar de los paralelos entre los dos autores, existen diferencias importantes.

Ortega dirige su atención al amor como un sentimiento interpersonal y se centra principalmente en las elecciones de amor. Scheler, por otro lado, tiene una visión general del amor que busca captar el fenómeno en todas sus formas. Esta diferencia de concepciones también se expresa en las características que ambos autores atribuyen al amor. Para Scheler, un signo de amor es que, sin importar cuánto dure realmente, plantea el reclamo de la eternidad o el infinito. Ortega, sin embargo, señala repetidamente la naturaleza fugaz del amor y afirma que es un fenómeno excepcional. Según Ortega, el signo de amor es la fidelidad al destino del amado, no importa cuán trágico y difícil sea. Además, la concepción de Ortega del libro de Lukács de 1913 Las tres etapas del erotismo (Die drei stufen der Erotik) se acuñó y formó antes de la idea contenida en el mismo, que el amor se enmarca como sentimiento en un contexto histórico. Esta perspectiva histórica, que a veces nos parece evidente como lectores del siglo XXI, no se encontró en ese momento en otros autores como Scheler o Pfänder. Finalmente, los puntos de vista de Ortega y Scheler difieren en la conexión entre el amor y los valores. Mientras que en Scheler los hombres descubren los valores gracias al amor, Ortega esto es posible en el hombre a través de su razón vital.

\subsubsection{Xirau: el amor y la percepción de los valores}

. Otro autor que contribuyó a la recepción del pensamiento de Scheler en España en esta primera fase es Joaquín Xirau. Xirau desarrolla una teoría del amor y la percepción de los valores en L'amor i la percepció dels valors y especialmente en Amor y Mundo, que está en sintonía con Scheler, pero que hasta ahora ha recibido poca atención. ${ }^{70}$ Xirau sigue las ideas de Scheler sobre el amor y el odio, siendo este último asociado fuertemente por Xirau con el resentimiento. Xirau considera que el amor es tan esencial para la naturaleza humana que incluso afirma que ignorarlo conllevaría a la desaparición de la misma historia, la literatura, el arte, la filosofía y la religión: "Nuestra vida

${ }^{69}$ Durán M., "Dos filósofos de la simpatía y el amor: Ortega y Max Scheler", en Revista La Torre, 1956, p. 114.

${ }^{70}$ El libro Amor y Mundo, fue traducido al alemán como Liebe und Welt, en el 2007 por Charlotte Frei. 
espiritual se desarrolla completamente en la esfera del amor."71 Metodológicamente, trabaja muy cerca de la fenomenología definiendo primero el amor ex negativo y luego extrayendo sistemáticamente sus características esenciales. Después de examinar diferentes concepciones del amor en el paganismo, el cristianismo y el positivismo, desarrolla su propia contribución, que está explícitamente orientada a Scheler. Xirau pregunta: “¿Qué es el amor?"72 y, en primer lugar, señala el significado múltiple de esta palabra, que se refiere tanto a los diferentes fenómenos llamados amor como a las múltiples cualidades en las que éste se produce (amor paternal, amor maternal, amor de pareja, amor a la ciencia, etc.). ${ }^{73} \mathrm{El}$ amor no se basa en el deseo sexual, el deseo, la simpatía, la contemplación desinteresada o la compasión, aunque estos fenómenos sí pueden acompañar al amor. ${ }^{74}$

Después de este acotamiento, Xirau llega a definir el amor como una "actitud radical de conciencia y vida", ${ }^{75}$ caracterizada por cuatro rasgos: En primer lugar, el amor presupone la plenitud de la vida interior, ya que el amor siempre debe ser una entrega. Además, el amor permite descubrir ciertos valores en la persona amada, de modo que aquí se habla de una "transformación radical de la realidad en el amor". ${ }^{76}$ Esta transformación consiste, Xirau siguiendo entonces a Scheler y Ortega y Gasset, en enfatizar los valores de la persona amada, que de lo contrario permanecería inclinada en una actitud u odio indiferente. No se trata de inventar "valores" en el amante que no están allí, sino de situarlo en lo más alto y ver sus valores. El amor no es una respuesta a los valores, porque incluso podemos amar a las personas con desvalorizaciones. Tercero, el amor implica "ilusión"77 y en ambos significados de la palabra en español: como deformación y transformación o como anticipación, deseo y anhelo. La última característica del amor, según Xirau, es que presupone "reciprocidad". Eso no debe entenderse en el sentido de una supresión de uno mismo en el amor, o una "fusión, confusión o abolición de límites", 78 porque el amor debe preservar la individualidad del sujeto. Declaraciones similares

\footnotetext{
${ }^{71}$ Xirau J., Amor y Mundo y otros escritos, Península, Barcelona,1983, p. 15.

${ }^{72}$ Ibidem, p. 89.

${ }^{73}$ Ibidem, p. 119.

${ }^{74}$ Ibidem, pp. 90, 94, 114, 118.

${ }^{75}$ Ibidem, p. 90

${ }^{76}$ Ibidem, p. 98.

${ }^{77}$ El término "Ilusión" es una peculiaridad del idioma español. Marías ha estudiado el fenómeno, dedicando su propio capítulo al amor. Cfr. Marías, J., Breve tratado de la ilusión, Alianza, Madrid, 2006.

${ }^{78}$ Ibidem, p. 108.
} 
también se pueden encontrar en Scheler. ${ }^{79}$ Xirau entiende el amor como el núcleo de la personalidad y se refiere a la idea de que cada persona se caracteriza por una nota de amor específica para él. Estas consideraciones apuntan a la inclusión del término de Scheler Ordo Amoris, el cual también se puede encontrar en Ortega y Gasset.

Xirau también se dedica al tema del odio en su trabajo. Interesante en este contexto es que Xirau casi usa el odio y el resentimiento como sinónimos, ${ }^{80}$ mostrando así la influencia de la concepción de resentimiento de Scheler. La tesis de Xirau es que ambos fenómenos no son antípodas simétricas. Scheler y Kolnai también sugirieron esta asimetría. ${ }^{81}$ Xirau afirma, pues, que el amor incluye el odio, mientras que el resentimiento excluye el amor. ¿Significa esto que hay odio en el amor? Esto estaría en contra de la tesis de Kolnai de que el amor libre de odio es posible y pondría a Xirau cerca de las posiciones psicoanalíticas. ¿Son posibles el amor y el odio simultáneamente con el mismo objeto? La posibilidad de sentimientos ambivalentes también ha ocupado a otros fenomenólogos. ${ }^{82}$ En este sentido, Xirau cree que en el fenómeno del "enamoramiento" el amor y el odio se nutren mutuamente; especialmente porque el amor está asociado con la sexualidad y el deseo. Sin embargo, esta tesis Xirau no está explícitamente formulada y desarrollada, y el lector solo puede encontrar aquí espacio para su propia interpretación. En la posición de Xirau me parece implícita la tesis de una separación entre los diferentes tipos de amor, que tiene su tradición en la historia de la filosofía y la psicología. Por un lado, existe el amor como entrega al otro, que la tradición ha llamado amor benevolentiae. En este amor, el sujeto se entiende a sí mismo en comunión con el objeto o incluso trasciende su propio ego en su dirección. Por otro lado, según esta concepción, existe el amor como deseo, que a menudo caracteriza el fenómeno del enamoramiento y se puede encontrar en la tradición como el amor concuspitiae. Aquí el sujeto trata de poseer y conquistar el objeto, de modo que persiste una distancia entre los polos del sujeto y el objeto. Es esta distancia la que abre la posibilidad de odio. Sin embargo, esto sigue sin desarrollarse en el postilado de Xirau acerca de una inmediación entre el amor y el odio.

\footnotetext{
${ }^{79}$ Scheler, M. Esencia y formas de la simpatía, op. cit.

${ }^{80}$ Xirau, J., Amor y Mundo y otros escritos, op. cit., p. 105.

${ }^{81}$ Scheler, M., Ordo Amoris, op. cit., Kolnai, A., op. cit.

${ }^{82}$ Kolnai, A., op. cit.
} 
En resumen, se puede afirmar que la primera fase de la recepción de la antropología de Scheler en España parte de sus primeros textos. Sin duda podemos hablar de una recepción del pensamiento antropológico temprano de Scheler en España, en el que el amor y su conexión con los valores reciben una gran atención.

\section{Bibliografía}

Conill, J., 2005, "Die Phänomenologie bei Zubiri”, en San Martín, J., Phänomenologie in Spanien, Konigshausen and Neumann, Würzburg, pp. 43-56.

Durán M., 1956, "Dos filósofos de la simpatía y el amor: Ortega y Max Scheler", en La Torre, 4, pp. 15-16.

García Morente, M., 2002, Ensayos sobre el progreso, Encuentro, Madrid.

Henckmann, W., 1998, Max Scheler, C.H. Beck, Múnich.

Lasaga Medina J., 2003, José Ortega y Gasset, 1883-1955, Vida y Filosofía, Biblioteca, Madrid.

Lerin J., 2005, Recepción y desarrollo de la fenomenología en España, en San Martín, J., Phänomenologie in Spanien, Würzburg, Konigshausen and Neumann.

Leroux, H., 1993, "Sur quelques aspects de la réception de Max Scheler en France", Phänomenologische Forschungen, 28/29, pp. 332-356.

Marías, J., 1983, Ortega. Circunstancia y vocación, Revista de Occidente, Madrid.

—, 1991, Acerca de Ortega, Espasa-Calpe, Madrid.

Mateo, M. S., 1961, Los Valores y Max Scheler y Ortega, en Humanitas, Revista de la Facultad de Filosofía y Letras, Universidad Nacional de Tucumán, vol. IX, pp. 157-170.

Mora, A., 2005, Fenomenología en el exilio (I) o el Colegio de Barcelona, en San Martín, J., Phänomenologie in Spanien, Würzburg, Kœnigshausen and Neumann, pp. 288-290.

Mulligan, K., 2003, Actes i objectes. Una anàlisi de la fenomenologia realista. Anuari de la Societat Catalana de Filosofia, vol. 13, pp. 41-64.

Orringer, N., 1979, Ortega y sus fuentes germánicas, Gredos, 1979, Madrid.

Ortega y Gaset, J., 1947, Obras Completas, vol. IV, Revista de Occidente, Alianza Editorial, Madrid.

, 1957, Sobre el amor. Meditaciones, Verlag-Anstalt, Stuttgart.

— 1981, Investigaciones Psicológicas, Revista de Occidente, Madrid.

- 1983, El Tema de nuestro tiempo, en Obras Completas, vol. III, Alianza Editorial, Madrid.

Perenya Blasi, F., 2005, Fenomenología en Cataluña, en San Martín, J., Phänomenologie in Spanien, Konigshausen and Neumann, Würzburg, pp. 295-296.

Pintor-Ramos, A., 1993, Schelers Einfluß auf das Denken der spanischsprachigen Welt, Phänomenologische Forschungen, 28/29, pp. 314-331.

San Martin, J., 2005, Phänomenologie in spanien, Konigshausen and Neumann, Würzburg. 
Scheler, M. F., 1954, Der Formalismus in der Ethik und die materiale Wertethik, Neuer Versuch der Grundlegung eines ethischen Personalismus, en Gesammelte Werke vol. II, Francke, Berna-Múnich.

—, 1954, Vom Ewigen im Menschen, en Gesammelte Werke, vol. V, ed. Scheler M. Francke, Berna.

- 1957, Ordo amoris, en Gesammelte Werke, vol. X (Schriften aus dem Nachlass I: Zur Ethik und Erkenntnislehre, ed. Scheler, M. Francke, Berna, pp. 347-376.

__, 1973, Wesen und Formen der Sympathie, en Gesammelte Werke vol. VII, ed. Frings Manfred, S. Francke, Berna, pp. 9-258.

—_, 1976, Die Stellung des Menschen im Kosmos en Gesammelte Werke, vol. IX, Späte Schriften, ed. Frings Manfred, S. Francke, Berna-Múnich, pp. 7-73.

— , 1976, Idealismus-Realismus, en Gesammelte Werke vol. IX, Späte Schriften, ed. Frings Manfred, S. Francke, Berna-Múnich, pp. 183-207.

Voigtländer, E., 1933, Bemerkungen zur Psychologie der Gesinnungen, en Neue Munich Philosophical, Hg. E. Heller y Löw, Leipzig, pp. 143-164.

Xirau, J., 1983, Amor y Mundo y otros escritos, Península, Barcelona. 